\title{
Apc inactivation, but not obesity, synergizes with Pten deficiency to drive intestinal stem cell-derived tumorigenesis
}

\author{
Tahmineh Tabrizian1,2,*, Donghai Wang1,2,3,*, Fangxia Guan1,2,3, Zunju Hu1,2,3, \\ Amanda P Beck ${ }^{4}$, Fabien Delahaye ${ }^{5,6}$ and Derek M Huffman1,2,3 \\ 1Department of Molecular Pharmacology, Albert Einstein College of Medicine, Bronx, New York, USA \\ 2Institute for Aging Research, Albert Einstein College of Medicine, Bronx, New York, USA \\ 3Division of Endocrinology, Department of Medicine, Albert Einstein College of Medicine, Bronx, \\ New York, USA \\ ${ }^{4}$ Department of Obstetrics \& Gynecology and Women's Health, Albert Einstein College of Medicine, \\ Bronx, New York, USA \\ ${ }^{5}$ Department of Genetics, Albert Einstein College of Medicine, Bronx, New York, USA \\ ${ }^{6}$ Department of Pathology, Albert Einstein College of Medicine, Bronx, New York, USA \\ *(T Tabrizian and D Wang contributed equally to this work)
}

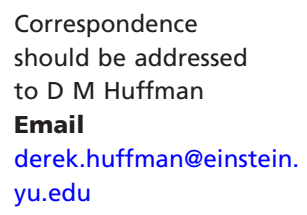

\begin{abstract}
Obesity is a major risk factor for colorectal cancer and can accelerate Lgr5+ intestinal stem cell (ISC)-derived tumorigenesis after the inactivation of Apc. However, whether non-canonical pathways involving PI3K-Akt signaling in ISCs can lead to tumor formation, and if this can be further exacerbated by obesity is unknown. Despite the synergy between Pten and Apc inactivation in epithelial cells on intestinal tumor formation, their combined role in Lgr5+-ISCS, which are the most rapidly dividing ISC population in the intestine, is unknown. Lgr5+-GFP mice were provided low-fat diet (LFD) or high-fat diet (HFD) for 8 months, and the transcriptome was evaluated in Lgr5+-ISCs. For tumor studies, Lgr5+-GFP and Lgr5+-GFP-Pten flox/flox mice were tamoxifen treated to inactivate Pten in ISCs and provided LFD or HFD until 14-15 months of age. Finally, various combinations of Lgr5+-ISC-specific, Apc- and Pten-deleted mice were generated and evaluated for histopathology and survival. HFD did not overtly alter Akt signaling in ISCS, but did increase other metabolic pathways. Pten deficiency, but not HFD, increased BrdU-positive cells in the small intestine $(P<0.05)$. However, combining $P$ ten and $A p c$ deficiency synergistically increased proliferative markers, tumor pathology and mortality, in a dose-dependent fashion $(P<0.05)$. In summary, we show that HFD alone fails to drive Akt signaling in ISCs and that Pten deficiency is dispensable as a tumor suppressor in Lgr5+-ISCs. However, combining Pten and Apc deficiency in ISCs synergistically increases proliferation, tumor formation and mortality. Thus, aberrant Wnt/ $/$-catenin, rather than PI3K-Akt signaling, is requisite for obesity to drive Lgr5+ ISCderived tumorigenesis.
\end{abstract}

\footnotetext{
Key Words

- colon cancer

- Pten

- obesity

- Lgr5

- Apc
}

๑ 2017 Society for Endocrinology Printed in Great Britain
Published by Bioscientifica Ltd.
Endocrine-Related Cancer (2017) 24, 253-265 


\section{Introduction}

Evidence suggests that intestinal stem cell(ISC) populations can serve as the origin of tumor development. Indeed, increased Wnt/ $\beta$-catenin signaling in Lgr $5+$, Bmi1+ or Lrig1+ ISCs, as well as $A h$-cre cells in the transit amplifying (TA) compartment, have been shown to rapidly promote tumorigenesis (Sangiorgi \& Capecchi 2008, Barker et al. 2009, Powell et al. 2014). The phosphoinositide-3-kinase (PI3K)-Akt pathway, which is commonly deregulated in human colorectal cancer, has been causally linked to tumor development in rodents, independent of canonical Wnt signaling, via PIK3CA mutations (Leystra et al. 2012) or Pten inactivation in the intestinal epithelium (Byun et al. 2011). Furthermore, constitutive activation of PI3K or loss of Pten in the intestinal epithelium can synergize with $A p c$ inactivation to dramatically increase intestinal tumor development (Shao et al. 2007, Marsh et al. 2008, Langlois et al. 2009, Deming et al. 2014). In small intestine, PTEN is most robustly expressed in the villus, but levels are also detectable in the crypt base, where Lgr5+-ISCs reside (Byun et al. 2011). However, to what extent the PI3K-Akt pathway may be involved in driving Lgr5+-ISC-derived tumorigenesis, either independently, or in cooperation with dysregulated Wnt/ $\beta$-catenin signaling, is unknown.

Obesity and diet strongly regulate CRC risk and progression (Kim et al. 2006, Pischon et al. 2006, Cheskin \& Prosser 2007, Giovannucci \& Michaud 2007, Bardou et al. 2013, Schlesinger et al. 2015) as well as intestinal tumor development (Gravaghi et al. 2008, Hata et al. 2011, Pettan-Brewer et al. 2011, Day et al. 2013, Huffman et al. 2013, Beyaz et al. 2016) in humans and rodent models, respectively. Even prior to tumor initiation, obesity appears to 'prime' the normal intestinal epithelium toward tumor development, by promoting proliferation of ISCs and hypertrophy of the epithelium (Mao et al. 2013), while also altering the epigenomic landscape of the colonic epithelium in a manner resembling cancer progression (Li et al. 2014). Obesity has also been shown to alter the systemic and local microenvironment in the gut, including the microbiome (Cani et al. 2008), which can create a pro-inflammatory environment in the colon to increase oxidative stress, genome instability and potential risk of CRC (O'Callaghan et al. 2009, Pendyala et al. 2011). More recently, diet-induced obesity was found to increase the number and function of Lgr5+ ISCs, while also promoting stemness and tumorigenicity of progenitor cells after inactivation of Apc (Beyaz et al. 2016). Although obesity can clearly instigate processes related to increased tumor risk, as well as accelerate intestinal tumorigenesis after loss of Apc in ISCs and epithelium (Gravaghi et al. 2008, Hata et al. 2011, Pettan-Brewer et al. 2011, Day et al. 2013, Huffman et al. 2013, Beyaz et al. 2016), whether obesity can promote Lgr5+-ISC-derived tumorigenesis via non-canonical mechanisms involving PI3K signaling (Huang \& Chen 2009, Vucenik \& Stains 2012) has not been investigated. Here, we demonstrate that although obesity can modify the transcriptome of Lgr5+-ISCs and expression of specific metabolic pathways, it fails to alter genes related to the Akt signaling and other proliferative pathways in Lgr5+-ISCs. Further, inactivation of Pten in Lgr5+ ISCs, either alone, or in combination with obesity, is insufficient to drive intestinal pathology and adenoma development in mice. However, we show Pten deletion in Lgr5+-ISCs can synergize with Apc loss to increase tumor multiplicity and worsen survival, demonstrating a previously unappreciated role for enhanced Akt-PI3K signaling, in cooperation with Apc deficiency, to drive Lgr5+-ISC-derived tumorigenesis.

\section{Materials and methods}

\section{Animals}

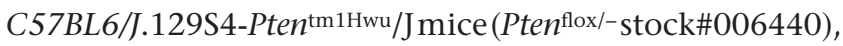
C57BL6/J.129P2-Lgr5tm1(cre/ERT2)Cle/J mice (Lgr5+-GFP; stock\#008875) and C57BL6;129S6-Gt(Rosa)26Sortm14(CAGtdTomato)Hze mice (Rosa26 ${ }^{\text {RFP }}$; stock\#007914) were all obtained from Jackson Laboratories. $A p c^{5805 / 5805}$ mice on a C57BL/6 background were a kind gift of Dr Leonard Augenlicht (Peregrina et al. 2015). To generate Pten deficiency and/ or $A p c$ deficiency in Lgr5+ ISCs, Lgr5+-GFP-Apc $c^{580 \mathrm{~S} /-}$ Pten flox/- mice were bred with $A p c^{5805 /-}-P_{t e n} n^{\text {flox/- }}$ animals to generate Lgr5+-GFP (Control), Lgr5+-GFP-Apc 580s/- (Apc Het), Lgr5+-GFP Pten ${ }^{\text {flox/flox }}\left(\right.$ Pten KO), Lgr5+-GFP-Apc $c^{580 \mathrm{~s} /-}$ $P_{\text {Pten }}^{\text {flox/flox }}$ (Apc het-Pten KO), Lgr5+-GFP-Apc $580 \mathrm{~s} / 580 \mathrm{~s}$ $(A p c \quad \mathrm{KO})$ and Lgr5+-GFP-Apc $580 \mathrm{~s} / 580 \mathrm{~s}-P t e n^{\text {flox/flox }} \quad(A p c$ KO-Pten KO) mice. Mice were genotyped as described (Barker et al. 2009, Byun et al. 2011, Peregrina et al. 2015), and males were weaned at 3 weeks of age and provided a purified low-fat diet (D12450H; Research Diets Inc, New Brunswick, NJ, USA). Animals were maintained under standard temperature and photoperiod as described (Huffman et al. 2013). All experimental procedures were approved by the Einstein Institutional Animal Care and Use Committee. 


\section{Experiment 1: Obesity and Lgr5+ intestinal stem cells}

At weaning, male Lgr5+-GFP mice were placed on a defined, purified ingredient LFD (3.85 kcal/g; D12450H). At 7-8 weeks of age, animals were randomized to remain on LFD $(n=6)$ or switched to a more energy-dense sucrosematched HFD feeding $(n=6)$ consisting of $45 \%$ Kcal from fat with lard as the predominant fat source in lieu of corn starch and maltodextrin, but all other components remained constant $(4.73 \mathrm{kcal} / \mathrm{g}$; D12451; Research Diets Inc) until $\sim 8$ months of age. At the end of the study, body weight was recorded, and animals were killed after a brief 3- to 4-h fast for blood collection, and isolation of Lgr5+ISCs from the small intestine by FACS, as described below, for RNA sequencing analysis.

\section{Experiment 2: Pten deficiency and obesity}

In order to determine the role of Pten deficiency on Lgr5+ISC-derived tumorigenesis under low-fat or high-fat-fed conditions, 3-months-old male Lgr5+-GFP (Control) and Lgr5+-GFP-Pten flox/flox (Pten KO) mice were injected intraperitoneally (i.p.) with $1 \mathrm{mg}$ tamoxifen (TAM) on two consecutive days to induce Cre recombinase in Lgr5+-ISCs. The efficacy of the TAM protocol to induce Cre recombination was confirmed in Lgr5+-GFP-Rosa reporter mice, as shown in Supplementary Fig. 2A and B (see section on supplementary data given at the end of this article). Animals were then placed on either a purified LFD or sucrose-matched HFD and monitored for up to 12 months after injection (15 months of age) for specimen collection and histopathology.

\section{Experiment 3: Pten and Apc deficiency}

It was reported that complete inactivation of $A p c$ in Lgr5+-ISCs led to rapid onset of intestinal adenomas and related mortality (Holik et al. 2014), whereas heterozygous deletion of Apc in Lgr5+ ISCs leads to significant pathology within 6 months of induction (Peregrina et al. 2015). In order to determine if Pten loss in Lgr5+-ISCs can synergize with $A p c$ inactivation, six groups of mice were generated (Lgr5+-GFP (control), Lgr5+-GFP-Apc $c^{500 /-}$ (Apc Het), Lgr5+-GFP-Pten flox/flox (Pten KO), Lgr5+-GFP-Apc $c^{580 \mathrm{~s} /-}$ Pten flox/flox (Apc het-Pten KO) Lgr5+-GFP-Apc $c^{5805 / 5805}(A p c$ KO), Lgr5+-GFP-Apc 580s/580s_Pten flox/flox (Apc KO-Pten KO)). Animals were injected with $1 \mathrm{mg}$ TAM on two consecutive days at 3-5 months of age as described previously and immediately placed on a purified diet (D12450H). Animals were then monitored for up to 4 months prior to killing, for tissue collection and histopathology ( $n=4-13 /$ group) and/or survival ( $n=9-13 /$ group). Mice were removed prior to 4 months after induction if $>25 \%$ weight loss was observed within a 1-week period, combined with signs of sickness and lethargy that suggested the animal was unlikely to survive an additional $24-48 \mathrm{~h}$ longer, and this was considered the time of death pending necropsy.

Plasma insulin and glucose determination Whole blood was collected from Lgr5+-GFP mice on LFD or HFD after a 3- to 4-h fast into K2-EDTA collection tubes (Sarstedt AG \& Co, Numbrect, Germany), and immediately centrifuged $\left(1500 \mathrm{~g} ; 4^{\circ} \mathrm{C}, 15 \mathrm{~min}\right)$ to separate plasma from red blood cells. Plasma Insulin levels were measured by a rat/mouse ELISA (EMD Millipore) with rat insulin standards using a spectrophotometer (Biorad iMark plate reader) following the manufacturer's instructions. Plasma glucose was determined via the glucose oxidase method with an Analox GM7 analyzer (Analox Inst., USA Inc, Lunenberg, MA, USA), as described previously (Muzumdar et al. 2009, Einstein et al. 2010, Huffman et al. 2016).

\section{Intestinal histopathology}

For evaluation of epithelial cell proliferation and migration in the small intestine, random mice were injected i.p. with $100 \mathrm{mg} / \mathrm{kg}$ BrdU (Sigma) $24 \mathrm{~h}$ prior to killing. At necropsy, the entire intestine was quickly excised, surrounding mesenteric fat was removed and the gut was divided into duodenum, jejunum, ileum and colon, as previously described (Huffman et al. 2013). Each segment was opened longitudinally, rinsed in icecold phosphate-buffered saline and carefully flattened for examination of tumor multiplicity with the aid of a dissecting magnifying lens. Macroadenomas ( > $0.5 \mathrm{~mm}$ diameter), when present, were counted in each segment of intestinal tissue and recorded. Tissue was subsequently rolled and fixed overnight in 10\% neutral-buffered formalin at $4^{\circ} \mathrm{C}$ for staging as a Swiss roll. Specimens were then processed through a series of alcohols and xylenes and embedded in paraffin. Hematoxylin \& eosin (H\&E)stained sections $(5 \mu \mathrm{m})$, from each segment of small intestine, capturing the entire proximal to distal length, were subsequently evaluated by a pathologist (A P B), who was blinded to the experimental groups, for histological changes following consensus recommendations for assessing intestinal pathology and tumors in rodents (Boivin et al. 2003).

Published by Bioscientifica Ltd. 


\section{D organoid assay}

Crypts were isolated from the small intestine of LFDand HFD-fed mice ( $n=4$ group) as described elsewhere (Yilmaz et al. 2012). Isolated crypts were washed with ADF medium, centrifuged at $300 \boldsymbol{g}$ for $5 \mathrm{~min}$, resuspended in ADF medium and counted on a hemocytometer. Approximately 250 crypts were then resuspended in $25 \mu \mathrm{L}$ of matrigel, transferred to a 48-well plate to solidify at $37^{\circ} \mathrm{C}$ for $30 \mathrm{~min}$ and overlaid with $250 \mu \mathrm{l}$ crypt culture medium (ADF 1×, Pen/Strep 1×, HEPES 1×, Glutamax 1×, N2 $1 \times$, B27 $1 \times$, N-acetyl-L-cysteine $1 \mu \mathrm{M}$, oggin $100 \mathrm{ng} /$ $\mathrm{mL}$, EGF $50 \mathrm{ng} / \mathrm{mL}$, Rock inhibitor $10 \mu \mathrm{M}$, and R-Spondin $500 \mathrm{ng} / \mathrm{mL}$ ) and maintained at $37^{\circ} \mathrm{C}$. Fresh medium was applied every 3 days and the number and area of budding crypts on day 5 and organoid formation on day 9 were assessed with a light microscope and normalized to the beginning number of counted crypts and expressed as organoids per crypt.

Immunohistochemistry Immunostaining was performed as previously described (Huffman et al. 2007, 2008). In brief, intestinal sections were subjected to antigen retrieval (Citrate buffer $\mathrm{pH}$ 6) using a pressure cooker on high steam for $10 \mathrm{~min}$. After rehydration, slides were treated with $3.0 \% \mathrm{H}_{2} \mathrm{O}_{2}$ for $5 \mathrm{~min}$ to quench endogenous peroxidase activity, subjected to an avidin-biotin blocking step (Vector Labs SP-2001) and subsequently blocked with pre-immune goat or rabbit serum (1\%) for $20 \mathrm{~min}$. Sections were then incubated with an antibody against Ki67 (1:400; cat\#12202) pAkt ${ }^{\text {Ser473 }}$ (1:50; cat\#4060), phospho- (Ser/Thr) Akt substrate (1:500; cat\#9611), $\beta$-catenin (1:100; cat\#8480) and antiBrdU antibody (1:200; cat\#5292) from Cell Signaling. A negative control was included in the same run using a subset of slides by omitting primary antibody from the staining procedure. Sections were then incubated with the appropriate biotinylated secondary antibody for $20 \mathrm{~min}$, followed by a streptavidin-HRP detection system (Vector) and application of 3,3'-diaminobenzidine (DAB) for visualization of the antigen-antibody complex (ScyTek). Digital files of all slides were then acquired with a PerkinElmer P250 High-Capacity Slide Scanner and positively stained cells were analyzed using QuantCenter Software.

\section{Flow cytometry}

Lgr5+-GFPHi-expressing cells were isolated from LFDand HFD-fed mice ( $n=6$ group) following established methods (36, 76). In brief, cells from isolated crypts were disassociated and subjected to Flow Cytometry (BD LSR II flow cytometer, Becton Dickinson, Franklin Lakes, NJ, USA) and analyzed with FlowJo software (Tree Star). Cells were gated to select for only Lgr5+-GFPHi-expressing ISCs and were sorted directly into TRIzol LS to ensure good RNA quality, prior to phenol/chloroform extraction. We routinely isolated $\sim 200,000-300,000$ Lgr5-GFPHi expressing ISCs per animal, and purity was confirmed by gene expression of Lgr5 in GFPHi-expressing cells vs GFPneg cells using established primers (Munoz et al. 2012).

\section{Total RNA library preparation and sequencing}

RNA sequencing libraries were prepared using the KAPA Stranded Rnaseq Kit with RiboErase (Kapa Biosystems) in accordance with the manufacturer's instructions. Briefly, 100 ng of total RNA was used for ribosomal depletion and fragmentation. Depleted RNA underwent first- and secondstrand cDNA synthesis and cDNA was adenylated, ligated to Illumina sequencing adapters and amplified by PCR (10 cycles). Final libraries were evaluated using fluorescentbased assays, including PicoGreen (Life Technologies), Qubit Fluorometer (Invitrogen) and Fragment Analyzer (Advanced Analytics) or BioAnalyzer (Agilent 2100). Deep sequencing ( $70 \mathrm{M}$ reads) was then performed on samples using an Illumina HiSeq2500 sequencer (v4 chemistry, v2 chemistry for Rapid Run) using $2 \times 50$-bp cycles.

\section{Expression analysis}

Reads were aligned to the mouse reference $10 \mathrm{~mm}$ using STAR aligner (v2.4.2a) (Dobin et al. 2013). Quantification of genes annotated in Gencode vM5 was performed using featureCounts (v1.4.3) and quantification of transcripts using Kalisto (doi:10.1038/nbt.3519). QC was collected with Picard (v1.83) and RSeQC (Wang et al. 2012) (http://broadinstitute.github.io/picard/). Normalization of feature counts was done using the DESeq2 package, version 1.10.1 (doi:10.1101/002832). Gene set enrichment was performed on genes found differentially expressed between HFD and LFD with a false discovery rate (FDR) of $5 \%$. Traditional gene set enrichment analysis does not take into account the physical characteristics of the gene and has been shown to be biased by factors such as the length of the gene (Geeleher et al. 2013). To address this, we used the Bioconductor package GoSeq (Young et al. 2010) developed to control for variability of length of genes to assess enriched pathways based on the KEGG database (Kyoto Encyclopedia of Genes and Genome). In addition,

Published by Bioscientifica Ltd 
one sample from the LFD group was determined to be a statistical outlier by PCA and was excluded from the analysis.

\section{Statistics}

Parametric data were analyzed by independent sample $t$-tests or two-way ANOVA, and longitudinal measures were assessed by repeated-measures ANOVA and planned contrasts were performed with Bonferroniadjustment when appropriate. Data were log transformed when necessary to ensure the normality of distribution. Non-parametric data were analyzed by the Kruskal-Wallis test and followed up with Mann-Whitney $U$ tests when appropriate. Survival analysis was performed using the Kaplan-Meier procedure and log-rank test. All analyses were performed using SPSS (SPSS). Experiments were designed to achieve $80 \%$ power to detect a mean difference of $-1.8(n=6)$ to $-1.5(n=8$ per group) with a standard deviation of 1.0 and alpha of 0.05. For RNAseq, the average expression and standard deviation for each group with $n=5-6$ samples per group for LFD and HFD respectively enabled $>80 \%$ power to detect at least one group expression difference $>2$ fold-change at an FDR $=0.05$. A $P \leq 0.05$ was considered statistically significant for all analyses.

\section{Results}

\section{HFD upregulates fatty acid metabolism, but not Akt pathway genes in Lgr5+-ISCs}

It was previously established that Akt-related genes are upregulated in the colonic mucosa in obese, tumor-prone mice (Pfalzer et al. 2016). To determine if the Akt pathway is upregulated in Lgr5+-ISCs with obesity in normal mice, we performed RNAseq on isolated Lgr5+ ISCs from LFDand HFD-fed animals. HFD mice were nearly $50 \%$ heavier than LFD mice (Fig. 1A; $P<0.01$ ), were hyperinsulinemic (Fig. 1B; $P<0.01$ ) and hyperglycemic (Fig. 1C; $P<0.001$ ). However, employing an ex vivo 3D intestinal organoid assay, previously used to demonstrate increased ISC proliferation by caloric restriction, rapamycin (Yilmaz et al. 2012), as well as obesity (Beyaz et al. 2016), we failed to observe any effect of HFD on ISC proliferation, as compared to LFD controls (Fig. 1D). Lgr5+-ISCs were next isolated by FACS, and purity was confirmed by qPCR (Fig. 1D and E). Transcriptome analysis by RNAseq on Lgr5+-ISCs detected 798 differentially regulated genes between LFD and HFD (adjusted $P$ value $\leq 0.05$; Supplementary Table 1 and Fig. $1 \mathrm{~F}$ and G). However, the magnitude of differences between differentially regulated genes between LFD and HFD were mostly limited and predominantly downregulated in HFD animals (Fig. 1G). To further characterize the overlap between our genes of interest and the Akt pathway, we ran a supervised analysis looking at the level of expression and distribution of $P$ values in our samples for genes annotated as part of the Akt pathway in KEGG database (Fig. 1H). Enrichment for a low $P$ value will indicate the correlation between diet and Akt pathway. Enrichment was quantified using the $\pi 1$ statistic (Storey \& Tibshirani 2003). Both, the clustering approach and the $\pi 1$ statistic confirmed the lack of enrichment for Akt pathway with a $\pi 1$ score equal to 0 and a random clustering of the samples (Fig. 1H). Likewise, no difference was observed for cancer, MAPK or Wnt pathways between groups (Supplementary Fig. 1). However, the unbiased analysis implicated several other pathways as differentially regulated, including an upregulation in fatty acid metabolism and PPAR signaling (Fig. 1I), with the latter consistent with a recent report (Beyaz et al. 2016).

\section{Pten inactivation alone, or combined with obesity, is insufficient to drive Lgr5+-ISC-derived tumorigenesis}

We next attempted to determine whether Pten serves as an obligate tumor suppressor in Lgr5+-ISCs and if tumorigenesis could be further augmented by obesity in Pten KO mice. Control and Pten KO male animals were i.p. injected with TAM at 3 months of age and placed on either a purified sucrose-matched LFD or a $45 \%$ HFD and monitored for up to 12 months after injection (1415 months of age). As expected, control and KO mice on HFD were heavier than their LFD-fed counterparts (Fig. 2A; $P<0.001$ ), whereas Pten deficiency in ISCs per se had no effect on body weight. Intestinal histopathology analysis in these mice revealed that neither HFD nor Pten deficiency per se in Lgr5+-ISCs profoundly altered gut pathology (Table 1). Of note, a reduction in multifocal crypt hyperplasia was observed in Pten KO mice on HFD $(P<0.05)$, but the pathologic relevance of this alteration is unknown as this histologic change was unrelated to the atypical form commonly linked to dysplasia and preneoplastic lesions. Sporadic dysplastic foci in the small intestine were identified in 2 LFD and HFD Pten KO mice, respectively, along with one instance of carcinoma and colonic dysplasia in a HFD-fed $\mathrm{KO}$ animal. However, the frequency of these alterations did not reach significance, whereas no macroadenomas were observed in these mice. Further, analysis of BrdU labeling in duodenum

Published by Bioscientifica Ltd. 

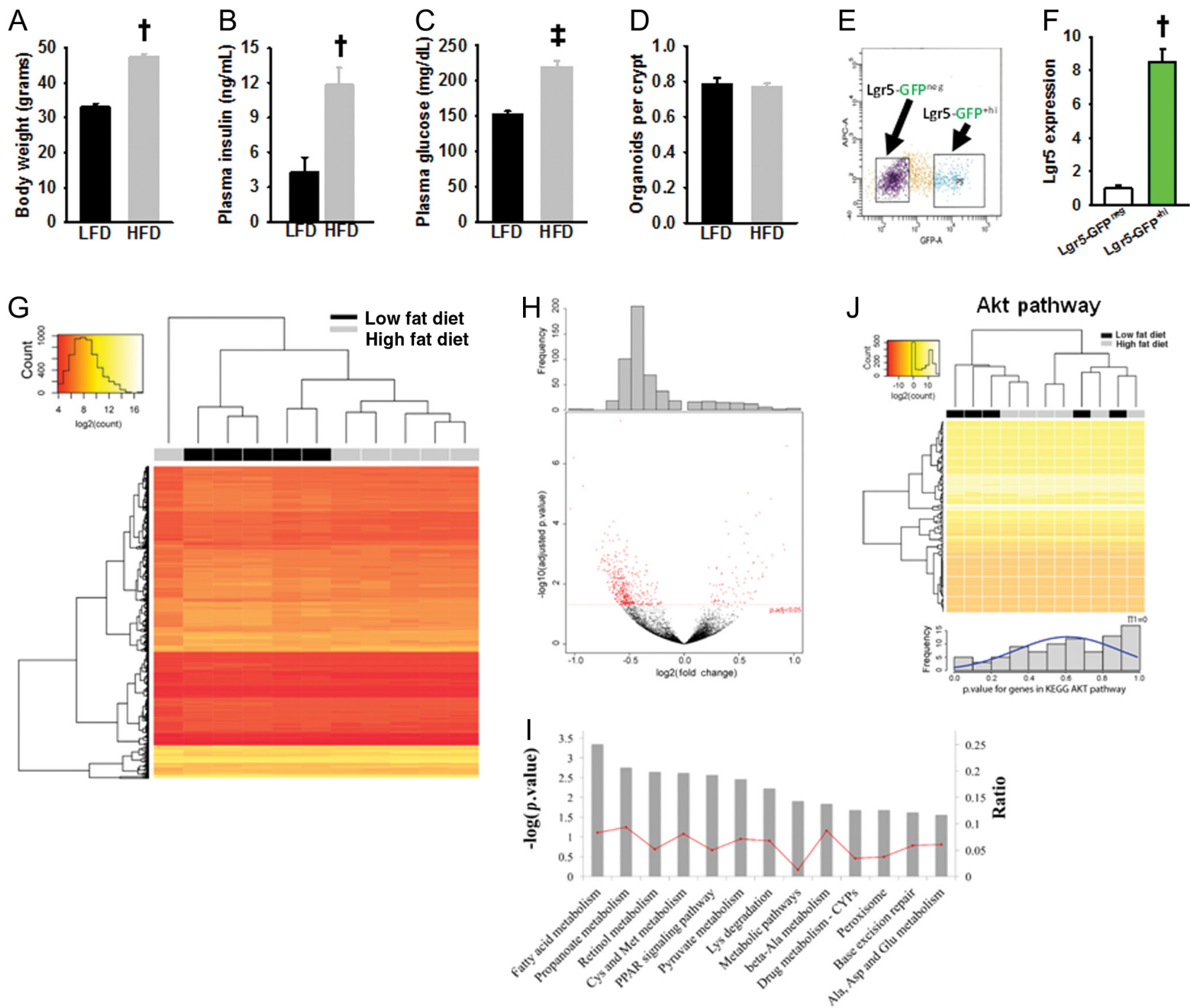

Figure 1

Characterization of the effect of high-fat vs low-fat diet on Lgr5+-ISC function and gene expression. (A) High-fat-fed mice weighed significantly more $(P<0.01),(B)$ and had elevated plasma insulin $(P<0.01)$ and glucose levels $(P<0.001)$ as compared to their low-fat-fed counterparts $(n=8$ per group). (C) Crypts isolated from high-fat and low-fat-fed mice small intestine did not demonstrate a significant difference in organoid formation at day 9 ( $n=4$ per group), which is expressed as counted organoids, normalized to the starting number of seeded crypts. (D) Lgr5+-GFPHi ISCs were isolated from LFD- $(n=5)$ and HFD-fed mice $(n=6)$ by FACS and, (E) expression of Lgr5 in the sorted cells were confirmed by qPCR $(P<0.01, n=6$ per group). (F) A total of 798 genes were differentially expressed when RNAseq analysis was performed on Lgr5+-ISC isolated from HFD- and LFD-fed mice $(P<0.05)$. (G) Of the differentially expressed genes between HFD and LFD, most were predominantly downregulated with HFD, as demonstrated by the volcano plot. (H) Akt pathway enrichment was studied using GoSeq and KEGG database, and no significant effect of diet was observed in the enrichment of Akt-related genes between HFD and LFD in isolated Lgr5+-ISCs. (I) Overall analysis showing the dominant differentially regulated pathways in Lgr5+-ISCs of low- and high-fat-fed mice. Interestingly, these pathways were all upregulated with HFD. Bars represent mean \pm S.E.M. Significantly different as compared to controls, ${ }^{\dagger} P<0.01, \mp P<0.01$.

revealed a significant effect of Pten KO $(P<0.001)$ and diet $(P<0.05)$ on proliferation, but no significant Pten $\times$ diet interaction was observed (Fig. 2B). Elsewhere, a main effect for Pten KO was observed in jejunum $(P<0.01)$ and ileum $(P<0.001)$, but diet had no effect on the number of BrdU-positive cells. An analysis of pAkt-positive staining revealed a significant main effect for Pten inactivation only in duodenum $(P=0.05)$, but no effect of diet or its interaction was observed, nor was any effect observed in other intestinal segments (Fig. 2C).

\section{Pten deficiency synergizes with Apc inactivation in Lgr5+ ISCs to drive tumorigenesis}

Despite the inability of Pten inactivation per se to drive ISC-derived tumorigenesis, Pten inactivation appears to

Published by Bioscientifica Ltd 
A

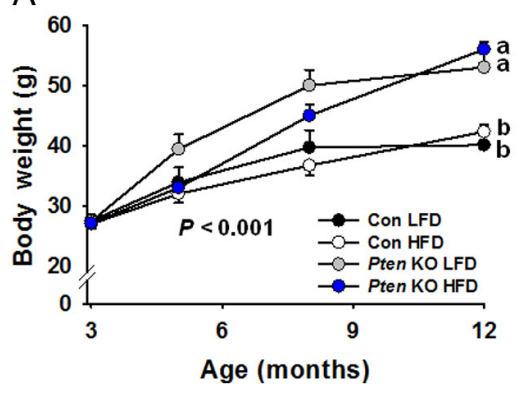

B

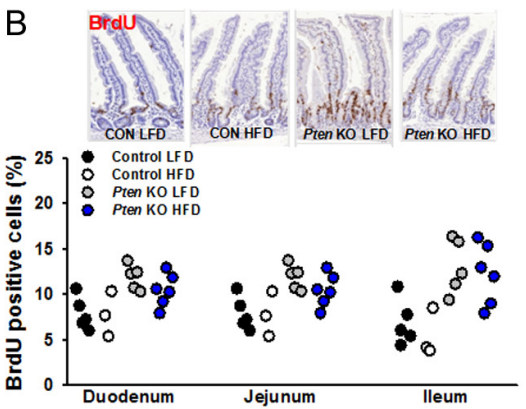

C

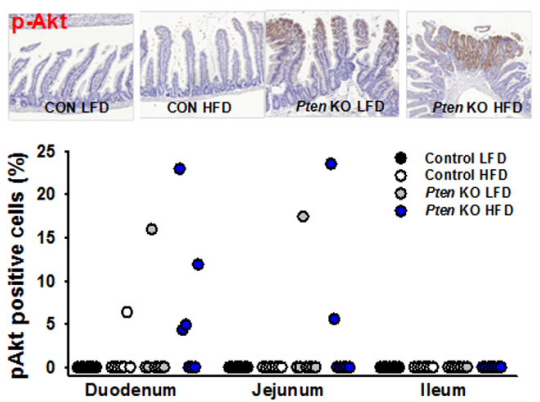

Figure 2

Effect of Pten deletion in Lgr5+-ISCs and obesity on ISC proliferation and Akt signaling in intestine. (A) Mice fed a high-fat diet gained significantly more body weight as compared to their low-fat-fed counterparts $(P<0.001)$ (con LFD $(n=8)$, Pten KO LFD $(n=9)$, con HFD $(n=14)$ and Pten KO HFD ( $n=17)$ ). Paraffin-embedded sections of small intestine from all 4 groups were immunostained for BrdU after a 24-h pulse. (B) Two-way ANOVA in duodenum revealed a significant main effect for Pten $\mathrm{KO}(P<0.001)$ and diet $(P<0.05)$, but no significant interaction. Similarly, a main effect for $P$ ten $\mathrm{KO}$ was observed in jejunum $(P<0.01)$ and ileum $(P<0.001)$, but no effect of diet or its interaction was observed (Con LFD $(n=7)$, Pten KO LFD $(n=5)$, Con HFD $(n=3)$, Pten KO HFD $(n=6))$ (C) Two-way ANOVA for pAkt-positive staining revealed a significant main effect for $P$ ten KO only in duodenum $(P=0.05)$, but no effect of diet or its interaction was observed on immunostaining for p-Akt-positive cells (con LFD ( $n=7)$, Pten KO LFD ( $n=7)$, Con HFD ( $n=7)$, Pten KO HFD $(n=7)$ ). Slides were scanned at $20 \times$, and histologic analysis was performed on 250 crypt-villi units in jejunum and ileum, and 100 crypt-villi units in duodenum, per sample in each independent experiment. Different letters denote a significant difference between groups, $P<0.01$.

influence ISC proliferation rates. Thus, we next determined if Pten inactivation can have additive or synergistic effects with $A p c$ deficiency on ISC-derived tumor development. To test this possibility, 6 unique models were generated, and all animals were TAM treated at 3-5 months of age and followed for up to 4 months for effects on tumor development and survival. Pten inactivation or Apc haploinsufficiency alone did not result in the development of pathology within 4 months (Table 1), whereas Apc HetPten KO mice developed a synergistic rise in dysplastic foci $(P<0.05)$ and macroadenoma formation (Fig. 3A and Table 2). Although mild pathology was observed in Apc KO mice, tumor multiplicity and pathology were markedly increased in Apc KO-Pten $\mathrm{KO}$ animals (Fig. 3A and Table 2; $P<0.05)$, with significant range in tumor multiplicity, which is a typical observation of aggressive, Apc-deficient tumor models (Gravaghi et al. 2008, Taketo \& Edelmann
2009, Huffman et al. 2013). Furthermore, no deaths were observed in WT, Pten KO, Apc Het or Apc KO mice over 16 weeks, with the latter observation contrary to prior reports of rapid mortality in Lgr5+-specific, Apc KO mice (Holik et al. 2014). Meanwhile, approximately $40 \%$ of $A p c$ Het-Pten KO mice did not survive up to 16 weeks, whereas $100 \%$ of Apc KO-Pten KO mice did not survive beyond 13 weeks after TAM induction.

\section{Pten and Apc deficiency selectively increases proliferative markers in intestine}

In order to determine the independent and combined effect of Apc and Pten inactivation in ISCs on proliferative markers in the intestine, we next stained for Ki67, $\beta$-catenin and pAkt. In duodenum, a significant main effect of $A p c$ deletion on Ki-67-positive cells was found

Table 1 Histopathology of the gastrointestinal tract in control and Lgr5+ stem cell specific Pten knockout mice fed either a low-fat or high-fat diet.

\begin{tabular}{|c|c|c|c|c|}
\hline & \multicolumn{2}{|c|}{ Low-fat diet } & \multicolumn{2}{|c|}{ High-fat diet } \\
\hline & Control $(n=8)$ & Pten KO $(n=9)$ & Control $(n=14)$ & Pten $\mathrm{KO}(n=17)$ \\
\hline Hyperplasia, crypt epithelial (focal) ${ }^{\dagger}$ & $0 \pm 0$ & $0 \pm 0$ & $0.14 \pm 0.14$ & $0.29 \pm 0.20$ \\
\hline Hyperplasia, crypt epithelial (multifocal) ${ }^{\dagger}$ & $1.28 \pm 0.18^{a}$ & $1.27 \pm 0.25^{a}$ & $1.07 \pm 0.47 a$ & $0.47 \pm 0.12^{b}$ \\
\hline Dysplastic foci ${ }^{\ddagger}$ & $0 \pm 0$ & $0.11 \pm 0.11$ & $0.28 \pm 0.13$ & $0.29 \pm 0.19$ \\
\hline Macroadenomas" & $0 \pm 0$ & $0 \pm 0$ & $0 \pm 0$ & $0 \pm 0$ \\
\hline Carcinomas $^{\ddagger}$ & $0 \pm 0$ & $0 \pm 0$ & $0 \pm 0$ & $0.06 \pm 0.06$ \\
\hline Dysplasia, colon & $0 \pm 0$ & $0 \pm 0$ & $0 \pm 0$ & $0.11 \pm 0.08$ \\
\hline
\end{tabular}

Data are means \pm S.E.M. Non-parametric data were analyzed by the Kruskal-Wallis non-parametric test planned contrast performed by Mann-Whitney $U$ test. A significant effect was observed for multifocal crypt hyperplasia (chi-square $=10.2 ; P=0.017$ ), with a significant reduction in Pten KO mice on HFD. Different letters denote a significant difference between groups, $P<0.05$.

\#Value based upon post-mortem analysis of total tumor multiplicity throughout the intestinal tract. Also shown in Fig. 2A. 'Value based on the

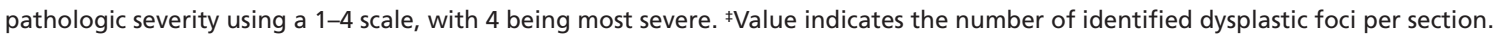


A

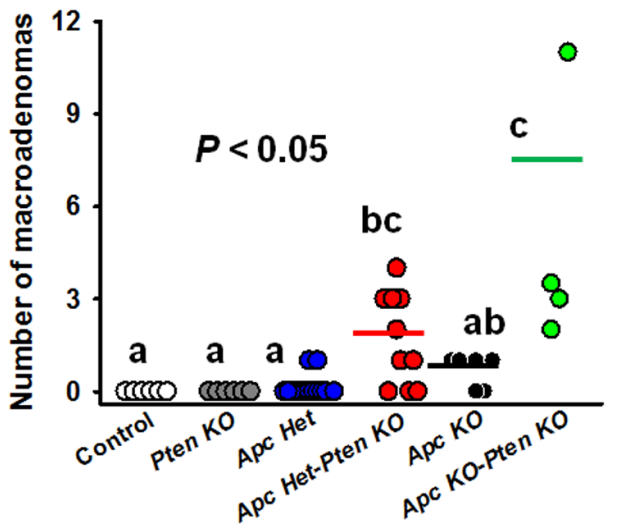

B

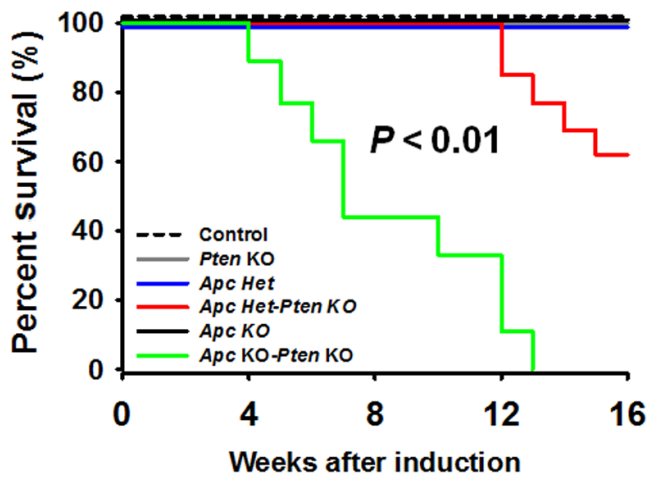

Figure 3

Pten deletion synergizes with Apc deficiency in Lgr5+-ISCs to promote tumorigenesis and worsen survival. Two-way ANOVA for tumor multiplicity revealed a significant main effect for Pten $(P<0.001)$, Apc Het $(P<0.01)$, Apc KO $(P<0.01)$, Pten $\times A p c$ Het $(P=0.01)$ and Pten $\times A p c$ KO interaction $(P=0.001)$. At necropsy, macroadenoma formation in the small intestine was absent in control and Pten KO mice, whereas a modest number of tumors were observed in $A p c$ Het and $A p c \mathrm{KO}$ animals. However, post hoc analyses confirmed that tumor multiplicity was significantly increased when combining Apc and Pten deficiency in a dose-dependent manner, such that Apc Het-Pten KO mice had increased tumor number, with the greatest evidence of tumor formation in Apc KO-Pten KO animals $(P<0.05)$ (con $(n=6)$, Pten KO $(n=8)$, Apc het $(n=13)$, Apc Het-Pten KO $(n=11)$, Apc KO $(n=6)$, $A p c$ KO-Pten KO (n=4)) (A). Likewise, although no death was observed in Apc Het or Apc KO mice, significant death began to be observed in $A p c$ Het-Pten KO animals within 3 months (60\% survival to 4 months), whereas a severe increase in mortality was observed in Apc KO-PTEN KO within 1 months, leading to $100 \%$ mortality within 3 months of TAM injection $(n=9-13$ group, $P<0.01)$ (Con $(n=6)$, Pten KO $(n=9), A p c$ het $(n=15)$, Apc Het-Pten KO $(n=13)$, Apc KO $(n=10)$ and Apc KO-Pten KO $(n=9))(B)$. For macroadenomas, different letters denote a significant difference between groups with Bonferroni adjustment $(P<0.05)$.

$(P<0.05)$, whereas in ileum, a main effect for Pten KO $(P<0.01)$, Apc KO $(P<0.01)$ and Pten $\times A p c$ Het interaction $(P<0.05)$ was observed, but no difference was observed between groups (Fig. 4A). Nuclear $\beta$-catenin localization, which is an indicator of dysregulated Wnt signaling, was unaffected by Pten or $A p c$ inactivation alone, but was significantly augmented by combined Pten and $A p c$ deficiency in duodenum and jejunum (Fig. 4B, $P<0.05$ ). Consistent with experiment 2, Pten deletion alone did not significantly increase cytoplasmic pAkt staining in intestine, nor was Akt activation affected by Apc loss per se, but staining was augmented when Pten inactivation was combined with $A p c$ haploinsufficiency or deficiency in duodenum and jejunum (Fig. $4 \mathrm{C}, P<0.05$ ). This was confirmed by immunostaining for Akt substrate in intestinal segments, which was lower in controls, but strong staining was present in Apc Het-Pten $\mathrm{KO}$ and Apc KO-Pten KO mice (Supplementary Fig. 3, $n=3$ per group).

\section{Discussion}

There has been an intense interest in both the origin and natural history of colorectal cancer onset and progression. Loss of $A p c$ has been viewed as an important initiating,

Table 2 Histopathology of the gastrointestinal tract in control and Lgr5+ stem cell specific Apc and Pten inactivation.

\begin{tabular}{|c|c|c|c|c|c|c|}
\hline & Control $(n=6)$ & Pten KO $(n=8)$ & $\begin{array}{c}\text { Apc Het } \\
(n=13)\end{array}$ & $\begin{array}{c}\text { Apc Het-Pten KO } \\
\qquad(n=11)\end{array}$ & Apc KO $(n=6)$ & $\begin{array}{c}\text { Apc KO-Pten KO } \\
(n=4)\end{array}$ \\
\hline Hyperplasia, crypt epithelial $\left(\right.$ focal) ${ }^{\dagger}$ & $0 \pm 0$ & $0.38 \pm 0.38$ & $0.23 \pm 0.21$ & $0.29 \pm 0.20$ & $1.50 \pm 0.50$ & $0 \pm 0$ \\
\hline $\begin{array}{l}\text { Hyperplasia, crypt epithelial } \\
\text { (multifocal) }^{\dagger}\end{array}$ & $0.67 \pm 0.18^{b}$ & $0.50 \pm 0.27^{b}$ & $0.61 \pm 0.17^{b}$ & $0 \pm 0^{a}$ & $0.67 \pm 0.49^{b}$ & $2.5 \pm 0.65 c$ \\
\hline Dysplasic foci ${ }^{\ddagger}$ & $0 \pm 0^{a}$ & $0 \pm 0^{a}$ & $0 \pm 0^{a}$ & $0.83 \pm 0.15^{b}$ & $1.00 \pm 0.21 \mathrm{ab}$ & $4.25 \pm 1.10 c$ \\
\hline Macroadenomas\# & $0 \pm 0^{a}$ & $0 \pm 0^{a}$ & $0.13 \pm 0.13^{a}$ & $0.38 \pm 0.15^{a}$ & $0 \pm 0^{a}$ & $2.0 \pm 0.4 \mathrm{~b}$ \\
\hline Carcinomas $^{\ddagger}$ & $0 \pm 0$ & $0 \pm 0$ & $0 \pm 0$ & $0.38 \pm 0.15$ & $0 \pm 0$ & $0.25 \pm 0.25$ \\
\hline Dysplasia, colon & $0 \pm 0$ & $0 \pm 0$ & $0.08 \pm 0.08$ & $0.13 \pm 0.10$ & $0 \pm 0$ & $0 \pm 0$ \\
\hline
\end{tabular}

Data are means \pm S.E.M. Non-parametric data were analyzed by the Kruskal-Wallis non-parametric test planned contrast performed by Mann-Whitney $U$. A significant effect was observed for multifocal crypt hyperplasia (chi-square $=17.8 ; P=0.003$ ), dysplastic foci (chi-square $=16.7 ; P=0.005$ ) and macroadenoma (chi-square $=27.8 ; P<0.001$ ). Different letters denote a significant difference between groups, $P<0.05$.

\#Value based upon post-mortem analysis of total tumor multiplicity throughout the intestinal tract. Also shown in Fig. $2 \mathrm{~A}$. ${ }^{\dagger} \mathrm{Value}$ based on the pathologic severity using a 1-4 scale, with 4 being most severe. ${ }^{\ddagger}$ Value indicates the number of identified dysplastic foci per section. 

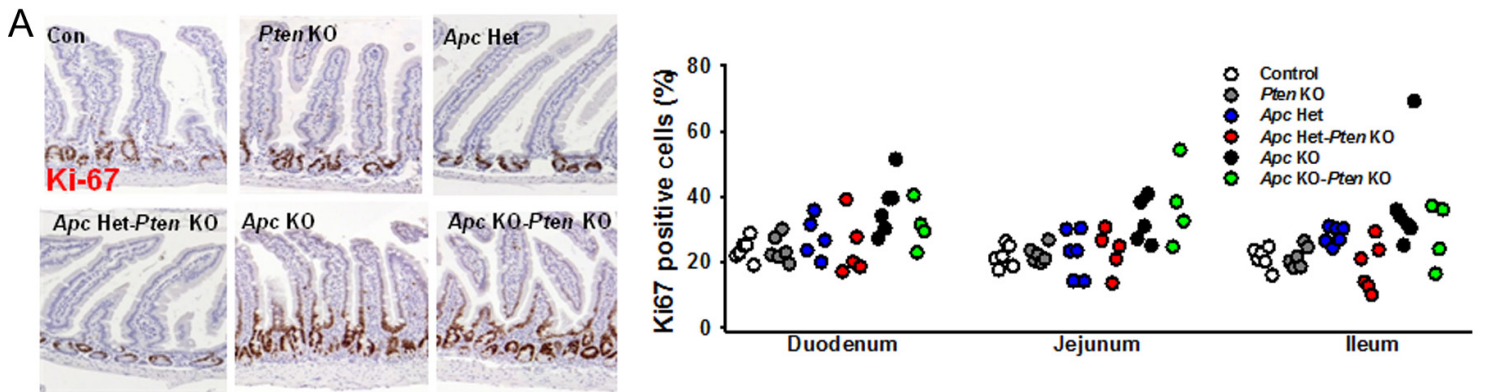

$\mathrm{B}$
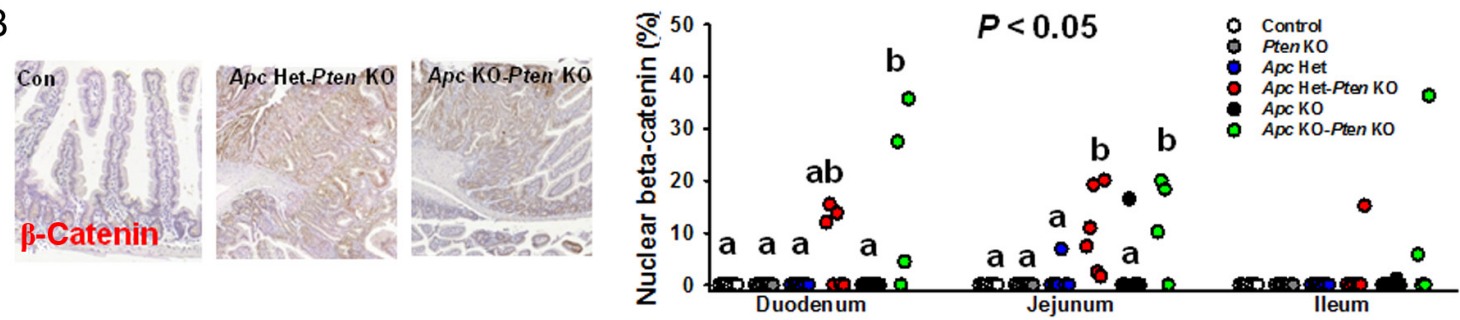

C
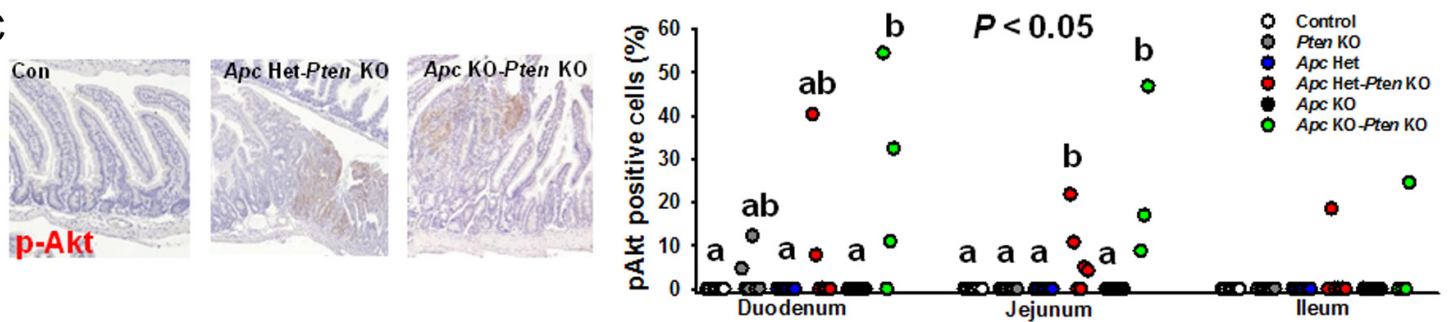

Figure 4

The effect of Pten and/or Apc deficiency in Lgr5+-ISCs on intestinal proliferative markers. Paraffin-embedded sections of small intestine in all 6 groups (Con $(n=6)$, Pten KO $(n=6)$, Apc het $(n=5-6)$, Apc Het-Pten KO $(n=5-6)$ Apc KO $(n=6)$ and Apc KO-Pten KO $(n=4))$ were immunostained for markers of Akt signaling and proliferation. (A) In duodenum, a significant main effect of $A p c$ deletion on Ki-67-positive cells was observed for $A p c K O(P<0.05)$, whereas in lleum, a main effect for Pten KO $(P<0.01)$, Apc KO $(P<0.01)$ and Pten $\times A p c$ Het interaction $(P<0.05)$ was observed, but no significant difference among groups was found. (B) Nuclear $\beta$-catenin localization, which is an indicator of dysregulated Wnt signaling, was significantly higher with combined Pten and $A p c$ deficiency in duodenum and jejunum, but not with Apc or Pten inactivation per se (duodenum main effect for $P$ ten KO $(P<0.01)$, Apc KO $(P<0.01)$ and $P$ ten $\times A p c$ KO interaction $(P<0.05)$, jejunum main effect for $P$ ten KO $(P<0.001), A p c$ Het $(P<0.01), A p c$ KO $(P<0.01)$, Pten $\times A p c$ Het $(P<0.05)$ and Pten $\times A p c$ KO interaction $(P<0.05))$. (C) Pten deletion alone failed to significantly increase cytoplasmic pAkt staining, but staining was augmented when combined with Apc haploinsufficiency or deficiency in duodenum and jejunum (duodenum main effect for $P$ ten KO $(P<0.01)$, Apc KO $(P<0.05)$ and Pten $\times A p c$ KO interaction $(P<0.05)$, jejunum main effect for $P$ ten KO $(P<0.001)$, Apc Het $(P<0.05), A p c$ KO $(P<0.01)$, $P$ ten $\times A p c$ Het $(P<0.05)$ and Pten $\times A p c$ KO interaction $(P<0.05))$. Slides were scanned at $20 \times$, and histologic analysis was performed on 250 crypt-villi units in jejunum and ileum and 100 crypt-villi units in duodenum, per sample in each independent experiment. Different letters denote a significant difference within an intestinal segment after Bonferroni adjustment, $P<0.05$.

and often required event in disease pathogenesis, with additional mutations in p53, Kras and the PI3K-Akt pathway among others, as necessary to promoting advanced disease (Huels \& Sansom 2015). Further, studies have demonstrated that Apc inactivation in several ISC populations can give rise to tumors (Sangiorgi \& Capecchi 2008, Barker et al. 2009, Powell et al. 2014), supporting the 'bottom-up' model of tumorigenesis, whereby dysplastic cells originate from ISCs in the crypt base rather than the villus. However, to what extent mutations affecting non-canonical signaling pathways such as the PI3K-Akt pathway in Lgr5+-ISCs can accelerate disease pathogenesis has not been studied.
Here, we demonstrate that in the absence of $A p c$ mutations, inactivation of Pten per se in Lgr5+-ISCs, either alone, or in combination with obesity, does not substantially alter intestinal homeostasis and is insufficient to drive tumorigenesis, suggesting that PTEN is dispensable as a tumor suppressor in these cells. This is in partial agreement with another report showing that Kras activation per se in Lgr5+-ISCs could result in hyperplasia, but failed to induce dysplasia or adenoma development (Feng et al. 2011). Given that Pten-knockout animals were not examined until 14-15 months of age, nearly 12 months after Cre induction, it is unlikely that the lack of observed transformation in these mice was

Published by Bioscientifica Ltd. 
confounded by the reportedly long latency of disease onset in Pten-deficient animals (Knobbe et al. 2008). Likewise, RNAseq confirmed high expression of PTEN in Lgr5+-ISCs (not shown), whereas insulin receptor, IGF-1 receptor expression, as well as insulin receptor substrates 1 and 2 were also present in these cells, suggesting that the insulin/IGF-1 signaling pathway and PTEN may be integral to cellular function in Lgr5+-ISCs.

Genetic and epigenetic alterations that lead to dysregulated PI3K-Akt signaling, including those affecting PTEN function, have been reported in human colon cancers (Liao et al. 2012, Ogino et al. 2014). However, in animal models, the effect of Pten inactivation in intestinal homeostasis has been somewhat controversial. Some reports observed that loss of Pten in the gut ( $\mathrm{He}$ et al. 2007, Byun et al. 2011, Yu et al. 2014) or whole body (Di Cristofano et al. 1998), is sufficient to drive intestinal pathology and tumorigenesis, but others have failed to observe any such effect of Pten loss alone on disease pathogenesis (Marsh et al. 2008, Langlois et al. 2009). The reason for these discrepant reports involving Pteninactivating mutations is not entirely clear, but may involve the diversity in promoters (Rosa, Ah-cre, Vil-cre, Vil-cre/ERT2), genetic backgrounds and time of follow-up (5, 50 days and 1 year) used. For instance, using the Vilcre mouse to delete Pten throughout the gut epithelium with 1-year follow-up (Langlois et al. 2009), resulted in hypertrophy and proliferation of the mucosa, but in contrast to prior reports (Di Cristofano et al. 1998), no evidence of tumors were detected. This contrary finding was speculated to potentially involve Pten deletion being restricted to the epithelium and not the stroma. However, a later report using the same Vil-cre promoter and follow-up period, but different genetic background, reported intestinal tumors in 19\% of mice (Byun et al. 2011). If intestinal tumors can in fact arise from ISCs after an inactivation mutation in Pten, our results suggest that Lgr5+ ISCs are unlikely to be the site of origin. However, we cannot rule out an effect of Pten loss in other epithelial cells, stromal cells as well as other ISC populations (Bmi1+, Lrig1+) or progenitor cells (TA cells) to instigate tumorigenesis.

Obesity per se has also been shown to increase intestinal proliferation (Mao et al. 2013), and data from our laboratory and others have shown that HFD can exacerbate tumorigenesis in several Apc models (Gravaghi et al. 2008, Hata et al. 2011, Pettan-Brewer et al. 2011, Day et al. 2013, Huffman et al. 2013), including Apcdeficient Lgr5+-ISCs (Beyaz et al. 2016). However, even when combined with obesity, Pten deficiency per se in Lgr5-ISCs, in the absence of Apc mutations, is insufficient to drive adenoma formation in these mice, even up to 15 months of age. Because spontaneous intestinal tumor development in normal mice is exceedingly rare, these data by no means suggest that obesity per se is not a risk factor for tumor development, but rather that additional mutations besides Pten (i.e. Apc) in ISCs are required for obesity-induced transformation and tumor progression in mice. Indeed, overwhelming evidence in humans has implicated obesity, and particularly visceral obesity, as a strong regulator of CRC risk and progression in humans (Kim et al. 2006, Pischon et al. 2006, Cheskin \& Prosser 2007, Giovannucci \& Michaud 2007, Bardou et al. 2013, Schlesinger et al. 2015), including greater risk of CRCrelated mortality (Calle et al. 2003).

Interestingly, in contrast to prior reports (Mao et al. 2013, Beyaz et al. 2016), we surprisingly did not observe any increase in ISC proliferation by obesity, as determined by intestinal organoid assay or BrdU-labeling studies, although caution should be exercised for results involving the latter assay, due to a limited sample size for control HFD mice. In addition, a transcriptome analysis of Lgr5+ ISCs from LFD- and HFD-fed mice did not detect any effect on growth and proliferation-related pathways (Akt, MAPK, Cancer and Wnt). Instead, the major pathways affected by obesity in these cells involved fatty acid metabolism, propanoate metabolism and PPAR signaling among others, with the latter observation consistent with a prior report implicating PPAR-delta in the effects of HFD on Lgr5+-ISCs (Beyaz et al. 2016).

A definitive explanation for some discordant results between our model and prior models is not entirely clear, but it is noteworthy that our study utilized a well-matched, purified and defined control companion diet for comparison, whereas many other studies have utilized standard rodent chow-based diets as a controlfeeding regimen. Although mice maintained on standard chow do remain leaner than their HFD counterparts, mouse chow also harbors a poorly defined concentration of macronutrients and micronutrients, as well as elevated fiber and vitamin $\mathrm{D}$, and the amount of these components can vary greatly among batches. Many of these constituents have important biologic activities, particularly in the gut, including effects on growth and differentiation pathways, the microbiome (Desai et al. 2016) and tumorigenesis (Augenlicht 2014). Thus, the inherent contribution of obesity in reports using chow as a control, particularly in the gut, should be interpreted 
with caution, given the stark differences in dietary composition between these formulas.

Given the evidence that Pten and Apc can synergize in the intestinal epithelium to promote tumorigenesis, we next performed a gene dosage experiment in Lgr5+ISCs by combining Pten deficiency with either Apc heterozygous or homozygous deletion. We observed a dose-dependent, synergistic rise in tumors along with accelerated mortality in double knockout mice. Indeed, although significant pathology and lethality were noted in combined Apc heterozygosity and Pten loss, observed effects on intestinal pathology and survival were far more extensive when combined with complete Apc inactivation in Lgr5+-ISCs. These findings are somewhat in agreement with other models that have found that inactivation of Apc or Pten throughout the intestinal epithelium augments Wnt/ $\beta$-catenin-driven tumor formation (He et al. 2007), though our results suggest far greater dysregulation when both genes are completely inactivated. The rapidity of disease onset and death in mice lacking Pten and ApC only in Lgr5+-ISCs was comparable to those reported by combined deficiency throughout the gut epithelium (Marsh et al. 2008), suggesting that Lgr5+-ISCs are a key site of tumor initiation by combined dysregulation of Wnt/ $\beta$-catenin and PI3K signaling. Likewise, the necessity for combined Pten and Apc loss to augment Akt activation was also consistent with prior observations in the intestine (Marsh et al. 2008). Collectively, these data confirm that PTEN alone is dispensable as a tumor suppressor in Lgr5+ISCs when Apc is present, whereas PTEN plays a tumorsuppressive role when Apc is lost in Lgr5+-ISCs.

In summary, we show that Pten loss per se in Lgr5ISCs is not required either as a tumor suppressor or for maintaining intestinal homeostasis when Apc is functional, even when combined with obesity. Further, obesity leads to modest alterations in the Lgr5+-ISC transcriptome and augments fatty acid-related pathways in Lgr5+-ISCs, but does not alter Akt signaling-related genes in these cells. In contrast, Pten loss per se in Lgr5+-ISCs, but not diet, explained alterations to intestinal proliferation and Akt signaling. Although $A p C$ inactivation was required to induce tumorigenesis in the intestine, disease severity and mortality were synergistically increased when this was further combined with Pten deficiency in Lgr5+-ISCs. Thus, these data demonstrate that Lgr5+-ISCs are an important site of Pten and Apc deficiency and establish the importance of PTEN in the control of PI3K/Akt signaling in these cells to prevent accelerated disease progression by canonical pathways involved in intestinal tumorigenesis.

\section{Supplementary data}

This is linked to the online version of the paper at http://dx.doi.org/10.1530/ ERC-16-0536.

\section{Declaration of interest}

The authors declare that there is no conflict of interest that could be perceived as prejudicing the impartiality of the research reported.

\section{Funding}

This work was supported by the NIA (R00AG037574, R56AG052981, P30AG038072), the Prevent Cancer Foundation, the American Institute for Cancer Research (AICR), the American Federation for Aging Research (AFAR) and Einstein Startup Funds to D M H. The authors would also like to acknowledge that experiments in the Einstein Analytical Imaging Core were supported by an NIH SIG award (\#1S10OD019961-01) and the $\mathrm{NCl}$ supported Einstein Cancer Center that provides partial support for the Flow Cytometry Core (P30CA013330).

\section{Acknowledgements}

The authors would also like to acknowledge Jinghang Zhang in the Einstein Flow Cytometry Core Facility for technical assistance, and the Einstein Computational Genomic Core for its support.

\section{References}

Augenlicht L 2014 Hidden effects of mouse chow. Science 346710. (doi:10.1126/science.346.6210.710-a)

Bardou M, Barkun AN \& Martel M 2013 Obesity and colorectal cancer. Gut 62 933-947. (doi:10.1136/gutjnl-2013-304701)

Barker N, Ridgway RA, van Es JH, van de Wetering M, Begthel H, van den Born M, Danenberg E, Clarke AR, Sansom OJ \& Clevers H 2009 Crypt stem cells as the cells-of-origin of intestinal cancer. Nature $\mathbf{4 5 7}$ 608-611. (doi:10.1038/nature07602)

Beyaz S, Mana MD, Roper J, Kedrin D, Saadatpour A, Hong SJ, BauerRowe KE, Xifaras ME, Akkad A, Arias E, et al. 2016 High-fat diet enhances stemness and tumorigenicity of intestinal progenitors. Nature 531 53-58. (doi:10.1038/nature17173)

Boivin GP, Washington K, Yang K, Ward JM, Pretlow TP, Russell R, Besselsen DG, Godfrey VL, Doetschman T, Dove WF, et al. 2003 Pathology of mouse models of intestinal cancer: consensus report and recommendations. Gastroenterology 124 762-777. (doi:10.1053/ gast.2003.50094)

Byun DS, Ahmed N, Nasser S, Shin J, Al-Obaidi S, Goel S, Corner GA, Wilson AJ, Flanagan DJ, Williams DS, et al. 2011 Intestinal epithelialspecific PTEN inactivation results in tumor formation. American Journal of Physiology: Gastrointestinal and Liver Physiology $\mathbf{3 0 1}$ G856-G864. (doi:10.1152/ajpgi.00178.2011)

Calle EE, Rodriguez C, Walker-Thurmond K \& Thun MJ 2003 Overweight, obesity, and mortality from cancer in a prospectively studied cohort of U.S. adults. New England Journal of Medicine 348 1625-1638. (doi:10.1056/NEJMoa021423)

Cani PD, Bibiloni R, Knauf C, Waget A, Neyrinck AM, Delzenne NM \& Burcelin R 2008 Changes in gut microbiota control metabolic endotoxemia-induced inflammation in high-fat diet-induced obesity and diabetes in mice. Diabetes 57 1470-1481. (doi:10.2337/db071403)
(C) 2017 Society for Endocrinology Printed in Great Britain
Published by Bioscientifica Ltd 
Cheskin LJ \& Prosser BJ 2007 Obesity and the risk of colon polyps. Journal of Clinical Gastroenterology 41 229-230. (doi:10.1097/01. mcg.0000248020.54256.b0)

Day SD, Enos RT, McClellan JL, Steiner JL, Velazquez KT \&Murphy EA 2013 Linking inflammation to tumorigenesis in a mouse model of high-fat-diet-enhanced colon cancer. Cytokine 64 454-462. (doi:10.1016/j.cyto.2013.04.031)

Deming DA, Leystra AA, Nettekoven L, Sievers C, Miller D, Middlebrooks M, Clipson L, Albrecht D, Bacher J, Washington MK, et al. 2014 PIK3CA and APC mutations are synergistic in the development of intestinal cancers. Oncogene 33 2245-2254. (doi:10.1038/onc.2013.167)

Desai MS, Seekatz AM, Koropatkin NM, Kamada N, Hickey CA, Wolter M, Pudlo NA, Kitamoto S, Terrapon N, Muller A, et al. 2016 A dietary fiber-deprived gut microbiota degrades the colonic mucus barrier and enhances pathogen susceptibility. Cell 167 1339.e1321-1353.e1321. (doi:10.1016/j.cell.2016.10.043)

Di Cristofano A, Pesce B, Cordon-Cardo C \& Pandolfi PP 1998 Pten is essential for embryonic development and tumour suppression. Nature Genetics 19 348-355. (doi:10.1038/1235)

Dobin A, Davis CA, Schlesinger F, Drenkow J, Zaleski C, Jha S, Batut P, Chaisson M \& Gingeras TR 2013 STAR: ultrafast universal RNA-seq aligner. Bioinformatics 29 15-21. (doi:10.1093/bioinformatics/bts635)

Einstein FH, Huffman DM, Fishman S, Jerschow E, Heo HJ, Atzmon G, Schechter C, Barzilai N \& Muzumdar RH 2010 Aging per se increases the susceptibility to free fatty acid-induced insulin resistance. Journals of Gerontology: Series A, Biological Sciences and Medical Sciences 65 800-808. (doi:10.1093/gerona/glq078)

Feng Y, Bommer GT, Zhao J, Green M, Sands E, Zhai Y, Brown K, Burberry A, Cho KR \& Fearon ER 2011 Mutant KRAS promotes hyperplasia and alters differentiation in the colon epithelium but does not expand the presumptive stem cell pool. Gastroenterology 141 1003-1013.e1001-1010. (doi:10.1053/j.gastro.2011.05.007)

Geeleher P, Hartnett L, Egan LJ, Golden A, Raja Ali RA \& Seoighe C 2013 Gene-set analysis is severely biased when applied to genomewide methylation data. Bioinformatics 29 1851-1857. (doi:10.1093/ bioinformatics/btt311)

Giovannucci E \& Michaud D 2007 The role of obesity and related metabolic disturbances in cancers of the colon, prostate, and pancreas. Gastroenterology 132 2208-2225. (doi:10.1053/j.gastro.2007.03.050)

Gravaghi C, Bo J, Laperle KM, Quimby F, Kucherlapati R, Edelmann W \& Lamprecht SA 2008 Obesity enhances gastrointestinal tumorigenesis in Apc-mutant mice. International Journal of Obesity 32 1716-1719. (doi:10.1038/ijo.2008.149)

Hata K, Kubota M, Shimizu M, Moriwaki H, Kuno T, Tanaka T, Hara A \& Hirose Y 2011 C57BL/KsJ-db/db-Apc mice exhibit an increased incidence of intestinal neoplasms. International Journal of Molecular Sciences 12 8133-8145. (doi:10.3390/ijms12118133)

He XC, Yin T, Grindley JC, Tian Q, Sato T, Tao WA, Dirisina R, PorterWestpfahl KS, Hembree M, Johnson T, et al. 2007 PTEN-deficient intestinal stem cells initiate intestinal polyposis. Nature Genetics 39 189-198. (doi:10.1038/ng1928)

Holik AZ, Young M, Krzystyniak J, Williams GT, Metzger D, Shorning BY \& Clarke AR 2014 Brg1 loss attenuates aberrant wnt-signalling and prevents wnt-dependent tumourigenesis in the murine small intestine. PLoS Genetics 10 e1004453. (doi:10.1371/journal. pgen.1004453)

Huang XF \& Chen JZ 2009 Obesity, the PI3K/Akt signal pathway and colon cancer. Obesity Reviews 10 610-616. (doi:10.1111/j.1467789X.2009.00607.x)

Huels DJ \& Sansom OJ 2015 Stem vs non-stem cell origin of colorectal cancer. British Journal of Cancer 113 1-5. (doi:10.1038/bjc.2015.214)

Huffman DM, Grizzle WE, Bamman MM, Kim JS, Eltoum IA, Elgavish A \& Nagy TR 2007 SIRT1 is significantly elevated in mouse and human prostate cancer. Cancer Research 67 6612-6618. (doi:10.1158/00085472.CAN-07-0085)
Huffman DM, Moellering DR, Grizzle WE, Stockard CR, Johnson MS \& Nagy TR 2008 Effect of exercise and calorie restriction on biomarkers of aging in mice. American Journal of Physiology: Regulatory, Integrative and Comparative Physiology 294 R1618-R1627. (doi:10.1152/ ajpregu.00890.2007)

Huffman DM, Augenlicht LH, Zhang X, Lofrese JJ, Atzmon G, Chamberland JP \& Mantzoros CS 2013 Abdominal obesity, independent from caloric intake, accounts for the development of intestinal tumors in $\mathrm{Apc}(1638 \mathrm{~N} /+)$ female mice. Cancer Prevention Research 6 177-187. (doi:10.1158/1940-6207.CAPR-12-0414)

Huffman DM, Farias Quipildor G, Mao K, Zhang X, Wan J, Apontes P, Cohen P \& Barzilai N 2016 Central insulin-like growth factor-1 (IGF1) restores whole-body insulin action in a model of age-related insulin resistance and IGF-1 decline. Aging Cell 15 181-186. (doi:10.1111/acel.12415)

Kim S, Baron JA, Mott LA, Burke CA, Church TR, McKeown-Eyssen GE, Cole BF, Haile RW \& Sandler RS 2006 Aspirin may be more effective in preventing colorectal adenomas in patients with higher BMI (United States). Cancer Causes and Control 17 1299-1304. (doi:10.1007/s10552-006-0075-x)

Knobbe CB, Lapin V, Suzuki A \& Mak TW 2008 The roles of PTEN in development, physiology and tumorigenesis in mouse models: a tissue-by-tissue survey. Oncogene 27 5398-5415. (doi:10.1038/ onc.2008.238)

Langlois MJ, Roy SA, Auclair BA, Jones C, Boudreau F, Carrier JC, Rivard N \& Perreault N 2009 Epithelial phosphatase and tensin homolog regulates intestinal architecture and secretory cell commitment and acts as a modifier gene in neoplasia. FASEB Journal 23 1835-1844. (doi:10.1096/fj.08-123125)

Leystra AA, Deming DA, Zahm CD, Farhoud M, Olson TJ, Hadac JN, Nettekoven LA, Albrecht DM, Clipson L, Sullivan R, et al. 2012 Mice expressing activated PI3K rapidly develop advanced colon cancer. Cancer Research 72 2931-2936. (doi:10.1158/0008-5472.CAN-114097)

Li R, Grimm SA, Chrysovergis K, Kosak J, Wang X, Du Y, Burkholder A, Janardhan K, Mav D, Shah R, et al. 2014 Obesity, rather than diet, drives epigenomic alterations in colonic epithelium resembling cancer progression. Cell Metabolism 19 702-711. (doi:10.1016/j. cmet.2014.03.012)

Liao X, Morikawa T, Lochhead P, Imamura Y, Kuchiba A, Yamauchi M, Nosho K, Qian ZR, Nishihara R, Meyerhardt JA, et al. 2012 Prognostic role of PIK3CA mutation in colorectal cancer: cohort study and literature review. Clinical Cancer Research 18 2257-2268. (doi:10.1158/1078-0432.CCR-11-2410)

Mao J, Hu X, Xiao Y, Yang C, Ding Y, Hou N, Wang J, Cheng H \& Zhang X 2013 Overnutrition stimulates intestinal epithelium proliferation through beta-catenin signaling in obese mice. Diabetes 62 3736-3746. (doi:10.2337/db13-0035)

Marsh V, Winton DJ, Williams GT, Dubois N, Trumpp A, Sansom OJ \& Clarke AR 2008 Epithelial Pten is dispensable for intestinal homeostasis but suppresses adenoma development and progression after Apc mutation. Nature Genetics 40 1436-1444. (doi:10.1038/ ng.256)

Munoz J, Stange DE, Schepers AG, van de Wetering M, Koo BK, Itzkovitz S, Volckmann R, Kung KS, Koster J, Radulescu S, et al. 2012 The Lgr5 intestinal stem cell signature: robust expression of proposed quiescent ' +4 ' cell markers. EMBO Journal 31 3079-3091. (doi:10.1038/emboj.2012.166)

Muzumdar RH, Huffman DM, Atzmon G, Buettner C, Cobb LJ, Fishman S, Budagov T, Cui L, Einstein FH, Poduval A, et al. 2009 Humanin: a novel central regulator of peripheral insulin action. PLOS ONE 4 e6334. (doi:10.1371/journal.pone.0006334)

O'Callaghan NJ, Clifton PM, Noakes M \& Fenech M 2009 Weight loss in obese men is associated with increased telomere length and decreased abasic sites in rectal mucosa. Rejuvenation Research 12 169-176. (doi:10.1089/rej.2008.0819) 
Ogino S, Lochhead P, Giovannucci E, Meyerhardt JA, Fuchs CS \& Chan AT 2014 Discovery of colorectal cancer PIK3CA mutation as potential predictive biomarker: power and promise of molecular pathological epidemiology. Oncogene 33 2949-2955. (doi:10.1038/ onc.2013.244)

Pendyala S, Neff LM, Suarez-Farinas M \& Holt PR 2011 Diet-induced weight loss reduces colorectal inflammation: implications for colorectal carcinogenesis. American Journal of Clinical Nutrition 93 234-242. (doi:10.3945/ajen.110.002683)

Peregrina K, Houston M, Daroqui C, Dhima E, Sellers RS \& Augenlicht LH 2015 Vitamin D is a determinant of mouse intestinal Lgr5 stem cell functions. Carcinogenesis 36 25-31. (doi:10.1093/carcin/bgu221)

Pettan-Brewer C, Morton J, Mangalindan R \& Ladiges W 2011 Curcumin suppresses intestinal polyps in APC Min mice fed a high fat diet. Pathobiology of Aging and Age Related Diseases 1 7013. (doi:10.3402/ pba.v1i0.7013)

Pfalzer AC, Kamanu FK, Parnell LD, Tai AK, Liu Z, Mason JB \& Crott JW 2016 Interactions between the colonic transcriptome, metabolome, and microbiome in mouse models of obesity-induced intestinal cancer. Physiological Genomics 48 545-553. (doi:10.1152/ physiolgenomics.00034.2016)

Pischon T, Lahmann PH, Boeing H, Friedenreich C, Norat T, Tjonneland A, Halkjaer J, Overvad K, Clavel-Chapelon F, Boutron-Ruault MC, et al. 2006 Body size and risk of colon and rectal cancer in the European Prospective Investigation Into Cancer and Nutrition (EPIC). Journal of the National Cancer Institute 98 920-931. (doi:10.1093/jnci/djj246)

Powell AE, Vlacich G, Zhao ZY, McKinley ET, Washington MK, Manning HC \& Coffey RJ 2014 Inducible loss of one Apc allele in Lrig1expressing progenitor cells results in multiple distal colonic tumors with features of familial adenomatous polyposis. American Journal of Physiology: Gastrointestinal and Liver Physiology 307 G16-G23. (doi:10.1152/ajpcell.00338.2014)
Sangiorgi E \& Capecchi MR 2008 Bmi1 is expressed in vivo in intestinal stem cells. Nature Genetics 40 915-920. (doi:10.1038/ng.165)

Schlesinger S, Lieb W, Koch M, Fedirko V, Dahm CC, Pischon T, Nothlings U, Boeing H \& Aleksandrova K 2015 Body weight gain and risk of colorectal cancer: a systematic review and meta-analysis of observational studies. Obesity Reviews 16 607-619. (doi:10.1111/ obr.12286)

Shao J, Washington MK, Saxena R \& Sheng H 2007 Heterozygous disruption of the PTEN promotes intestinal neoplasia in APCmin/+ mouse: roles of osteopontin. Carcinogenesis 28 2476-2483. (doi:10.1093/carcin/bgm186)

Storey JD \& Tibshirani R 2003 Statistical significance for genomewide studies. PNAS 100 9440-9445. (doi:10.1073/pnas.1530509100)

Taketo MM \& Edelmann W 2009 Mouse models of colon cancer. Gastroenterology 136 780-798. (doi:10.1053/j.gastro.2008.12.049)

Vucenik I \& Stains JP 2012 Obesity and cancer risk: evidence, mechanisms, and recommendations. Annals of the New York Academy of Sciences 1271 37-43. (doi:10.1111/j.1749-6632.2012.06750.x)

Wang L, Wang S \& Li W 2012 RSeQC: quality control of RNA-seq experiments. Bioinformatics 28 2184-2185. (doi:10.1093/ bioinformatics/bts356)

Yilmaz OH, Katajisto P, Lamming DW, Gultekin Y, Bauer-Rowe KE, Sengupta S, Birsoy K, Dursun A, Yilmaz VO, Selig M, et al. 2012 mTORC1 in the Paneth cell niche couples intestinal stem-cell function to calorie intake. Nature $\mathbf{4 8 6}$ 490-495. (doi:10.1038/ nature11163)

Young MD, Wakefield MJ, Smyth GK \& Oshlack A 2010 Gene ontology analysis for RNA-seq: accounting for selection bias. Genome Biology 11 R14. (doi:10.1186/gb-2010-11-2-r14)

Yu M, Trobridge P, Wang Y, Kanngurn S, Morris SM, Knoblaugh S \& Grady WM 2014 Inactivation of TGF-beta signaling and loss of PTEN cooperate to induce colon cancer in vivo. Oncogene 33 1538-1547. (doi:10.1038/onc.2013.102)

Received in final form 8 March 2017

Accepted 28 March 2017

Accepted Preprint published online 28 March 2017
(C) 2017 Society for Endocrinology Printed in Great Britain
Published by Bioscientifica Ltd 